\title{
Editorial: Mobile Elements and Plant Genome Evolution, Comparative Analyzes and Computational Tools
}

\author{
Ruslan Kalendar ${ }^{1,2 * t}$, Francois Sabot ${ }^{3 t}$, Fernando Rodriguez ${ }^{4 t}$, Gennady I. Karlov ${ }^{5 t}$, \\ Lucia Natali $^{6+}$ and Karine Alix ${ }^{7+}$ \\ ${ }^{1}$ HiLIFE Institute of Biotechnology, University of Helsinki, Helsinki, Finland, ${ }^{2}$ National Laboratory Astana, Nazarbayev \\ University, Nur-Sultan, Kazakhstan, ${ }^{3}$ DIADE, University of Montpellier, CIRAD, IRD, Montpellier, France, ${ }^{4}$ Josephine Bay Paul \\ Center for Comparative Molecular Biology and Evolution, Marine Biological Laboratory (MBL), Woods Hole, MA, \\ United States, ${ }^{5}$ Laboratory of Applied Genomics and Crop Breeding, All-Russia Research Institute of Agricultural \\ Biotechnology, Moscow, Russia, ${ }^{6}$ Department of Agriculture, Food and Environment, University of Pisa, Pisa, Italy, ${ }^{7}$ GQE - \\ Le Moulon, Université Paris-Saclay, INRAE, CNRS, AgroParisTech, Gif-sur-Yvette, France
}

Keywords: plant genome evolution, transposable elements (TE), retrotransposon (RTN), retrotransposon (RTN)based markers, transposon display (TD)

Edited and reviewed by: Chuang Ma,

Northwest $A$ and $F$ University, China

*Correspondence:

Ruslan Kalendar ruslan.kalendar@helsinki.fi

tORCID:

Ruslan Kalendar orcid.org/0000-0003-3986-2460 Francois Sabot orcid.org/0000-0002-8522-7583 Fernando Rodriguez orcid.org/0000-0003-4044-8734 Gennady I. Karlov orcid.org/0000-0002-9016-103X Lucia Natali orcid.org/0000-0003-3179-5910 Karine Alix orcid.org/0000-0002-8133-0743

Specialty section:

This article was submitted to Plant Systems and Synthetic Biology, a section of the journal Frontiers in Plant Science

Received: 02 July 2021 Accepted: 30 August 2021 Published: 24 September 2021

Citation:

Kalendar R, Sabot F, Rodriguez F, Karlov Gl, Natali L and Alix K (2021) Editorial: Mobile Elements and Plant

Genome Evolution, Comparative Analyzes and Computational Tools.

Front. Plant Sci. 12:735134.

doi: 10.3389/fp/s.2021.735134

\section{Editorial on the Research Topic}

Mobile Elements and Plant Genome Evolution, Comparative Analyzes and Computational Tools

\section{TRANSPOSABLE ELEMENTS TURN OUT TO BE FUNCTIONAL}

Multiple changes that occur constantly in the plant genome allow an organism to develop from a single-celled embryo to a multicellular organism. A significant part of these changes is associated with the recombination activity of numerous classes of interspersed repeats. These numerous families of interspersed repeats were often called "junk DNA" as first, they were not associated with any vital protein-coding processes. Now, more and more clues indicate that such repeated DNA might play major roles in the genome as functional "non-coding" DNA (Pennisi, 2012; Ariel and Manavella, 2021). Transposable elements (TEs), such as DNA transposons and retrotransposons, are the main part of these interspersed repeats (Vitte et al., 2014). The diverse families of retrotransposons are highly abundant genetic elements that are related to retroviruses (Wicker et al., 2007). Although retrotransposons are not "true" mobile elements like DNA transposons - from the comparison of their transposition mechanisms, retrotransposable elements (RTEs) are notably constitutive of heterochromatin and form a variety of major chromosomal structures such as centromeres (Bennetzen and Wang, 2014) and represent the main intergenic part of the plant genome (Kalendar et al., 2020). RTE mobility is ensured through an RNA intermediate, allowing a Copy-And-Paste approach for their transposition. Their own-encoded RNA is reverse transcribed using their own (or not) encoded enzymes, that will re-create from the single-strand RNA matrix a double-strand DNA at a new location. This reverse transcription can be either through extra-chromosomal DNA within a nucleocapsid (e.g., and implies RT/RNAseH + INT enzymes for LTR retrotransposons) or directly at the insertion site (e.g., Target-Prime Reverse Wicker et al., 2007). Such Copy-And-Paste mechanisms allow a quick invasion of naive genomes, and is responsible for massive genome increase in a few periods of time (Piegu et al., 2006). Such invasions belong to a few number of initial active copies, called Master copies; however, while these copies must be transcriptionally active (i.e., able to produce RNA), they can be translationally inactive and may rely on other copies for enzymatic activities (Sabot and Schulman, 2006). When transcription mechanism, with only the RT as a minimal set of enzymes for LINEs and SINEs; 
recently inserted, neo-copies can be related phylogenetically because of their sequence identity; subsequent mutations (point mutation, recombination, and so on) will then occur and their lineage can then be complex to recompose. Once inserted, each copy has its own evolutionary history and some may acquire biological role (or some part of the copy) and being excepted by their host. Some RTEs can provide evolutionary advantages to the host and were demonstrated to play a significant role in plant adaptation (Song and Cao, 2017). If the fact that TEs can be beneficial to the host is now accepted, recent advances in the field has placed RTEs at the center of the current debate on eukaryotic and notably plant evolution. To advance this important research field, in the Research Topic "Mobile Elements and Plant Genome Evolution, Comparative Analyses, and Computational Tools" we focused on the efficiency of new genomic tools for the discovery of TEs, and highlighted some recent studies on the role of mobile elements in the evolution of the host genome, as well as on genome-wide comparative analysis and profiling of transposable elements.

\section{MOBILE ELEMENT AND HOST GENOME EVOLUTION}

Different retrotransposon families, each with its own lineage and structure, may have been active at distinct phases in the evolution of a species. Retrotransposon sequences bear the promoters that bind the nuclear factors of transcription initialization and initiate RNA synthesis by polymerases II or III. In the article entitled "Additional ORFs in Plant LTR-Retrotransposons" by Vicient and Casacuberta, LTR-retrotransposons that carry additional but not retrotransposon-specific open reading frames (aORF) were discovered and analyzed. This discovery reinforces the unique potential of LTR-retrotransposons as evolutionary tools, thanks to their ability to provide new genetic variants within a genome. The presence of antisense aORFs in characterized LTR-retrotransposon families from various angiosperm species indicates a putative role of these coding regions in the transposition process. To clarify the functions of these aORFs, further analysis of TE transcript splicing variants and thus access to the TE functional diversity would be very valuable.

\section{TRANSPOSABLE ELEMENTS AS DRIVERS OF STRUCTURAL AND FUNCTIONAL VARIATIONS}

Thanks to their remarkable diversity in size, genomic organization and transposition process, TEs are important drivers of genome evolution and species diversity (Vicient and Casacuberta, 2017). Keidar-Friedman et al. and Bariah et al. reported the potential impact of miniature transposable element insertions on the expression of genes in different wheat species, with the articles entitled "The Evolutionary Dynamics of a Novel Miniature Transposable Element in the Wheat Genome" and "Where the Wild Things Are: Transposable Elements as Drivers of Structural and Functional Variations in the Wheat Genome." TE-mediated genomic rearrangements and insertions of mobile genetic elements in gene-rich regions may act as mutagens and contribute to genome alteration (Vicient and Casacuberta, 2017). TE-mediated epigenetic modifications lead to phenotypic diversity, genetic variation, and environmental stress tolerance. Potential TEs also contribute to genome plasticity and have a dramatic impact on the genetic diversity and evolution of the wheat genome. Using transposon display (Kalendar et al., 2021) and genome-wide profiling analysis of insertional polymorphisms of transposable elements (Kalendar and Schulman, 2006), the authors discovered large genomic rearrangement events, such as deletions and introgressions in the wheat genome. Highthroughput bioinformatics with next-generation sequencing (NGS) were key tools in these studies (Vondrak et al., 2020). Structural rearrangements, gene duplications, and variation in TE contents may have a large impact on the overall genomic structure and may be responsible for phenotypic diversity (Belyayev et al., 2010). In the article entitled "Genome Size Variation and Comparative Genomics Reveal Intraspecific Diversity in Brassica rapa," Boutte et al. investigated structural variants and changes in the repetitive fraction between two accessions of Brassica rapa, and characterized genome-size variation among a core collection, using comparative genomics and cytogenetic approaches. Detection of large genomic variants between different cultigroups of the same species B. rapa highlighted the potential impact of the differential insertion dynamics of repeated elements in the intraspecific variability of genome size and chromosomal structure. This study also justifies the recent and general effort to construct pangenomes when access to the genomic diversity of a given species is sought.

TEs contribute also to the driving force in the evolution of epigenetic regulation and have a long-term impact on genomic instability and evolution. Remnants of RTEs appear to be overrepresented in transcription regulatory modules and other regions conserved among distantly related species, which may have implications for our understanding of their impact on speciation. In the article entitled "Sequencing Multiple Cotton Genomes Reveals Complex Structures and Lays Foundation for Breeding," Pan et al. revealed that post-polyploidization of cotton genome instability resulted in numerous genomic structural changes that were accompanied by the expansion of LTR-retrotransposon families with an unbalanced contribution from the constituent genomes of cotton, with TEs from the D-genome being more active than TEs from the A-genome. This study also demonstrated that TE transposition activity coincided with cotton genome divergence, supporting the major role of TEs in genome shaping and speciation. Finally, the authors depicted the evolutionary past of cotton plants, which were recursively affected by polyploidization, like all plant lineages (Alix et al., 2017): a decaploidization contributed to the formation of the genus Gossypium prior to the wellknown neo-tetraploidization that accompanied the formation of the currently widely cultivated cotton species. The resulting functional innovation comes directly from the diversity of duplicated genes, as suggested by their uncommonly high evolutionary rates. 


\section{CHROMOSOME EVOLUTION WITH TRANSPOSABLE ELEMENTS AND SATELLITE DNAS}

The centromere is the unique part of the chromosome that combines a highly conserved function with a large variability of its constituent DNA sequences. In the article entitled "Functional Allium fistulosum centromeres comprise arrays of a long satellite repeat, insertions of retrotransposons and chloroplast DNA" Kirov et al. studied the functional centromere organization in the large-sized chromosomes of the species Allium fistulosum and A. cepa. They demonstrate that long and high-copy repeats are associated with insertions of retrotransposons and chloroplast DNA sequences, with tandem repeats being also constitutive of the centromeres. This article provides an insight into the major role of repetitive DNA in centromere function. Among evolutionary factors, repetitive sequences play multiple roles notably in sex chromosome evolution. As such, the Spinacia genus serves as an ideal model to investigate the evolutionary mechanisms underlying the transition from homomorphic to heteromorphic sex chromosomes. This was studied in the article entitled "Genome-Wide Analysis of Transposable Elements and Satellite DNAs in Spinacia Species to Shed Light on Their Roles in Sex Chromosome Evolution" by Li et al. Major repetitive

\section{REFERENCES}

Alix, K., Gerard, P. R., Schwarzacher, T., and Heslop-Harrison, J. S. P. (2017). Polyploidy and interspecific hybridization: partners for adaptation, speciation and evolution in plants. Ann. Bot. 120, 183-194. doi: 10.1093/aob/mcx079

Ariel, F. D., and Manavella, P. A. (2021). When junk DNA turns functional: transposon-derived non-coding RNAs in plants. J. Exp. Bot. 72, 4132-4143. doi: 10.1093/jxb/erab073

Belyayev, A., Kalendar, R., Brodsky, L., Nevo, E., Schulman, A. H., and Raskina, O. (2010). Transposable elements in a marginal plant population: temporal fluctuations provide new insights into genome evolution of wild diploid wheat. Mobile DNA 1:6. doi: 10.1186/1759-8753-1-6

Bennetzen, J. L., and Wang, H. (2014). The contributions of transposable elements to the structure, function, and evolution of plant genomes. Annu. Rev. Plant Biol. 65, 505-530. doi: 10.1146/annurev-arplant-050213-035811

Kalendar, R., Raskina, O., Belyayev, A., and Schulman, A. H. (2020). Long tandem arrays of cassandra retroelements and their role in genome dynamics in plants. Int. J. Mol. Sci. 21:2931. doi: 10.3390/ijms21082931

Kalendar, R., and Schulman, A. (2006). IRAP and REMAP for retrotransposonbased genotyping and fingerprinting. Nat. Protocols 1, 2478-2484. doi: 10.1038/nprot.2006.377

Kalendar, R., Shustov, A., and Schulman, A. (2021). Palindromic sequence-targeted (PST) PCR, version 2: an advanced method for high-throughput targeted gene characterization and transposon display. Front. Plant Sci. 12:691940. doi: 10.3389/fpls.2021.691940

Pennisi, E. (2012). Genomics. ENCODE project writes eulogy for junk DNA. Science 337, 1159-1161. doi: 10.1126/science.337.6099.1159

Piegu, B., Guyot, R., Picault, N., Roulin, A., Sanyal, A., Kim, H., et al. (2006). Doubling genome size without polyploidization: dynamics of retrotransposition-driven genomic expansions in Oryza australiensis, a wild relative of rice. Genome Res. 16, 1262-1269. doi: 10.1101/gr.5290206

Sabot, F., and Schulman, A. H. (2006). Parasitism and the retrotransposon life cycle in plants: a hitchhiker's guide to the genome. Heredity 97, 381-388. doi: $10.1038 /$ sj.hdy. 6800903 sequence classes were identified in male and female genomes of Spinacia species and their ancestral relative sugar beet in order to decipher the evolutionary processes of sex chromosome evolution using NGS data. The differences of repetitive DNA sequences correlate with the formation of sex chromosomes and satellite DNAs are highly accumulated at the sex determination locus, demonstrating the role of repetitive DNA in sex chromosome evolution.

\section{AUTHOR CONTRIBUTIONS}

RK, FS, and KA prepared the draft. All authors listed have made a substantial, direct and intellectual contribution to the work, and approved it for publication.

\section{FUNDING}

This work was supported by the Science Committee of the Ministry of Education and Science of the Republic of Kazakhstan in the framework of program funding for research (AP08855353).

\section{ACKNOWLEDGMENTS}

We thank all authors and reviewers for their contributions to this special issue and the support of the editorial office.

Song, X., and Cao, X. (2017). Transposon-mediated epigenetic regulation contributes to phenotypic diversity and environmental adaptation in rice. Curr. Opin. Plant Biol. 36, 111-118. doi: 10.1016/j.pbi.2017.02.004

Vicient, C. M., and Casacuberta, J. M. (2017). Impact of transposable elements on polyploid plant genomes. Ann. Bot. 120, 195-207. doi: 10.1093/aob/mcx078

Vitte, C., Fustier, M. A., Alix, K., and Tenaillon, M. I. (2014). The bright side of transposons in crop evolution. Brief Funct. Genom. 13, 276-295. doi: 10.1093/bfgp/elu002

Vondrak, T., Avila Robledillo, L., Novak, P., Koblizkova, A., Neumann, P., and Macas, J. (2020). Characterization of repeat arrays in ultra-long nanopore reads reveals frequent origin of satellite DNA from retrotransposon-derived tandem repeats. Plant J. 101, 484-500. doi: 10.1111/tpj.14546

Wicker, T., Sabot, F., Hua-Van, A., Bennetzen, J. L., Capy, P., Chalhoub, B., et al. (2007). A unified classification system for eukaryotic transposable elements. Nat. Rev. Genet. 8, 973-982. doi: 10.1038/nrg2165

Conflict of Interest: The authors declare that the research was conducted in the absence of any commercial or financial relationships that could be construed as a potential conflict of interest.

Publisher's Note: All claims expressed in this article are solely those of the authors and do not necessarily represent those of their affiliated organizations, or those of the publisher, the editors and the reviewers. Any product that may be evaluated in this article, or claim that may be made by its manufacturer, is not guaranteed or endorsed by the publisher.

Copyright (c) 2021 Kalendar, Sabot, Rodriguez, Karlov, Natali and Alix. This is an open-access article distributed under the terms of the Creative Commons Attribution License (CC BY). The use, distribution or reproduction in other forums is permitted, provided the original author(s) and the copyright owner(s) are credited and that the original publication in this journal is cited, in accordance with accepted academic practice. No use, distribution or reproduction is permitted which does not comply with these terms. 